An official journal of the / Un journal officiel de la "Société Sénégalaise de Cancérologie" (SOSECAN)

Journal homepage: www.africanjournalofoncology.com

Original article / Article original

DOI: https://doi.org//0.54266/ajo.I.I.28.28

\title{
Treatment of endometrial cancer at the Joliot Curie Institute in Dakar: about 30 cases
}

\section{Traitement des cancers de l'endomètre à l'institut Joliot Curie de Dakar : à propos de 30 cas}

\author{
J. Thiam", E. Kahatwa', S. Ka', MM. Dieng', D. Diouf', AC. Diallo', PM. Gaye², A. Dem'.
}

I Institut Joliot Curie de Dakar, Sénégal.

2 Service de radiothérapie, Hôpital Dalal Jamm, Sénégal.

\begin{abstract}
AIM: To study the therapeutic aspects of endometrial cancer at the Joliot Curie Institute in Dakar. MATERIALS AND METHODS: This is a retrospective study conducted at the Joliot Curie Institute in Dakar including all cases of endometrial cancer confirmed by histology between January 2012 and December 2016. RESULTS: There were 30 cases of endometrial cancer over a period of five years. Endometrioid adenocarcinoma was the most common type of histology and was found in 25 patients, or $84 \%$ of cases. The patients were classified as stages I and II of FIGO in $43 \%$ of cases, stage III in $24 \%$ of cases and stage IV in $33 \%$ of cases. Colpo-hysterectomy with pelvic lymphadenectomy $(\mathrm{CHL})$ was the main surgical procedure performed. External radiotherapy was performed in three patients or $10 \%$. A case of radiation cystitis and radiodermatitis were recorded as a radiotherapy complication. No patient received brachytherapy. Chemotherapy was performed in 17 patients, ie $56.6 \%$. She was adjuvant in 10 patients, or $34 \%$ of cases. Pelvic recurrences were noted in three patients who underwent $10 \%$ surgery. After 13 months of follow-up, survival was $50 \%$. CONCLUSION: The most effective treatment modality for endometrial cancer at the Joliot Curie Institute was surgery followed by chemotherapy and Radiation therapy.

KEYWORDS: Cancer; Endometrium; Surgery; Chemotherapy; Radiotherapy.
\end{abstract}

OBJECTIF : Etudier les aspects thérapeutiques des cancers de l'endomètre à l'Institut Joliot Curie de Dakar. MATERIELS ET METHODES : II s'agit d'une étude rétrospective menée à l'Institut Joliot Curie de Dakar incluant tous les cas de cancers d'endomètre confirmés par l'histologie entre Janvier 2012 et Décembre 2016. RESULTATS : II s'agissait de 30 cas de cancers d'endomètre sur une période de cinq ans. L'adénocarcinome endométrioïde était le type histologique le plus fréquent et était retrouvé chez 25 patientes soit $84 \%$ des cas. Les patientes étaient classées stades I et II de FIGO dans $43 \%$ des cas, stade III dans $24 \%$ des cas et stade IV dans 33\% des cas. La colpo-hystérectomie avec lymphadénectomie pelvienne (CHL) était le principal geste chirurgical effectué. Une radiothérapie externe était effectuée chez trois patientes soit $10 \%$. Un cas de cystite radique ainsi qu'une radiodermite étaient enregistrés comme complication liée à la radiothérapie. Aucune patiente n’a bénéficié de curiethérapie. La chimiothérapie était effectuée chez 17 patientes soit $56,6 \%$. Elle était adjuvante chez 10 patientes soit $34 \%$ des cas. Les récidives pelviennes étaient notées chez trois patientes opérées soit 10\%. Après 13 mois de suivi, la survie était de $50 \%$. CONCLUSION : Le traitement du cancer de l'endomètre à l'Institut Joliot Curie de Dakar est marqué par la prépondérance de la chirurgie qui est encadré par la chimiothérapie et/ou la radiothérapie externe.

MOTS-CLES : Cancer ; Endomètre ; Chirurgie ; Chimiothérapie ; Radiothérapie.

\section{INTRODUCTION}

Le cancer de l'endomètre est le premier cancer gynécologique de la femme en occident [I]. Sa particularité dans les pays en développement est plus liée à son stade avancé au diagnostic qu'à ses facteurs de risques [2, 3]. Ces stades avancés associés à la rareté ou l'inconstance de la radiothérapie externe et de la curiethérapie expliquent le contexte difficile de prise en charge. L'objectif de ce travail était d'étudier les aspects thérapeutiques du cancer de l'endomètre à l'Institut Joliot Curie de Dakar.

\section{MATERIELS ET METHODES}

Nous avons mené une étude rétrospective sur une période de cinq ans allant de janvier 2012 à décembre 2016 à l'Institut Joliot Curie de Dakar. Tous les cas de cancers d'endomètre prouvés par une hystéroscopie suivie d'une histologie ou prouvés sur l'analyse histologique d'une pièce opératoire étaient pris en compte. Les paramètres étudiés étaient les stades de la maladie, le traitement et le pronostic. La collecte des données a été effectuée sur un fichier Excel@ puis exportée sur Epi InfoC pour analyse.

\section{RESULTATS}

Nous avions retrouvé 30 cas de cancers d'endomètre sur une période de cinq ans soit une moyenne de six cas par an.

L'histologie était obtenue avant la chirurgie chez 10 patientes soit $33,3 \%$ des cas grâce à l'hystéroscopie et le curetage de l'endomètre. Toutes les autres patientes (77\%) avaient eu une histologie après la chirurgie. L'adénocarcinome endométrioïde était le type histologique le plus fréquent et était retrouvé chez 25 patientes soit $84 \%$ des cas.

Les patientes étaient classées stades I et II de FIGO dans 43\% des cas, stade III dans $24 \%$ des cas et stade IV dans 33\% des cas. 
La colpo-hystérectomie avec lymphadénectomie pelvienne (CHL) était le principal geste chirurgical effectué et se retrouvait chez 14 patientes (56\%). Deux patientes présentant un adénocarcinome non endométrioïde ont eu une chirurgie régionale. Un geste limité à la biopsie était réalisé chez une patiente présentant un cancer de l'endomètre au stade IVB avec carcinose diffuse et ascite (Tableau I).

Tableau I : Répartition des gestes chirurgicaux chez les patientes présentant un cancer de l'endomètre

\begin{tabular}{|c|c|c|c|}
\hline Geste & & Nombre & Pourcentage (\%) \\
\hline $\mathrm{CHL}$ & & 14 & 56 \\
\hline $\begin{array}{l}\text { Hystérectomie } \\
\text { annexectomie }\end{array}$ & et & 7 & 28 \\
\hline Chirurgie régionale & & 2 & 8 \\
\hline Biopsie & & l & 4 \\
\hline Hystérectomie seule & & 1 & 4 \\
\hline Total & & 25 & 100 \\
\hline
\end{tabular}

CHL : colpo-hystérectomie avec lymphadénectomie

Cinq patientes opérées soit $20 \%$ présentaient des résidus macroscopiques. En per-opératoire, quatre incidents ont été rapportés, il s'agissait de deux cas d'ouverture accidentelle de la vessie, soit $8 \%$ des cas, un cas de section de l'uretère, soit $4 \%$ des cas, et un cas de plaie de la veine iliaque externe lors du curage, soit $4 \%$ des cas. En post-opératoire, une patiente, soit $4 \%$ des cas, avait présenté une éviscération.

Une radiothérapie externe était effectuée chez trois patientes soit $10 \%$. Cette radiothérapie était adjuvante chez deux patientes et palliative chez une patiente. La dose administrée était de 42,5 Gy en adjuvant et de $60 \mathrm{~Gy}$ en palliative. Un cas de cystite radique ainsi qu'une radiodermite étaient enregistrés comme complication liée à la radiothérapie (7\% des cas). Aucune patiente n'a bénéficié de curiethérapie. La chimiothérapie était effectuée chez 17 patientes, soit $56,6 \%$. Elle était adjuvante chez 10 patientes, soit $34 \%$ des cas. Les récidives pelviennes étaient notées chez trois patientes opérées (10\% des cas). Le délai moyen d'apparition des récidives était de sept mois avec des extrêmes de deux à 12 mois. Chez quatre patientes (13,3\%) l'évolution était marquée par l'apparition d'une récidive métastatique. Le siège de la métastase était péritonéal chez deux patientes se traduisant par une ascite et une carcinose, hépatique et péritonéal chez une autre patiente et pleuro pulmonaire pour la dernière. A 13 mois de suivi, $50 \%$ des patients étaient vivantes.

\section{DISCUSSION}

Le traitement du cancer de l'endomètre dépend des facteurs pronostiques et du stade. L'évaluation du stade dépend localement de l'imagerie par résonance magnétique (IRM) avec injection de produit de contraste qui est l'examen de référence [4]. Elle est supérieure à l'échographie et la tomodensitométrie dans l'évaluation pré-thérapeutique et l'extension myométriale, cervicale et ganglionnaire locorégionale des cancers de l'endomètre [5]. Sa prescription est limitée par la rareté des machines et le coût de sa réalisation dans notre pratique. Le grade histo-pronostique est uniquement appliqué à l'adénocarcinome de type I, le type II étant considéré d'emblée de haut grade [6]. Nous avons retrouvé plus de cas d'adénocarcinomes endométrioïdes. Dans les types II, le gade n'est pas toujours précisé par les pathologistes ce qui rend difficile la prise de décision thérapeutique. La règle dans notre pratique est de faire un traitement d'emblée maximal.

La chirurgie est la pierre angulaire du traitement. Elle permet une meilleure stadification. Il subsiste des controverses sur son étendue, la voie d'abord et la place de la radiothérapie et de la chimiothérapie [7]. Dès le stade II, il est préconisé un curage lombo-aortique. Le risque de récidive aortique est élevé [8]. La voie d'abord laparoscopique est recommandée dans les stades précoces. Cependant tout stades confondus le taux de conversion est de $23 \%$ [9].

En dehors des stades I à faible risque de récidive (IA de type I et de grade histologique $\mathrm{I}$ et 2) qui ne nécessitent pas de traitement adjuvant, la radiothérapie post-opératoire est le traitement standard. Elle réduit de façon significative le risque de rechute locorégionale, elle n'améliore cependant pas la survie globale $[10,1 \mathrm{I}]$. Quoique la radio-chimiothérapie semble faire, de plus en plus, ses preuves en situation néoadjuvante pour les cancers localement avancés [12]. Les complications de la radiothérapie externe ne sont pas rares comme dans notre série d'autant plus que nous avons utilisé un appareil de radiothérapie externe utilisant du Cobalt sans conformation. Les récidives sont vaginales et métastatiques. Les récidives vaginales sont très symptomatiques. Elles sont le plus souvent curables si elles s'avèrent isolées. Les patientes récidivant de façon métastatique seraient symptomatiques dans $70 \%$ des cas. La réalisation d'un examen clinique rigoureux lors de chaque visite paraît indispensable [13]. Dans notre pratique, les récidives sont aussi diagnostiquées tardivement que les tumeurs primitives du fait du niveau de vie des populations et de l'analphabétisme.

Les taux de survie à 5 ans varient en fonction du stade de la maladie. Ils varient de $80 \%$ pour les stades I à $10 \%$ pour les stades IV [10, 14]. Les faibles taux de survie sont liés dans nos conditions aux stades avancés et à la difficulté d'accès à la radiothérapie et à la chimiothérapie [15].

\section{CONCLUSION}

Le carcinome de l'endomètre est un cancer de bon pronostic dans ses formes histologiques les plus habituelles et dans les stades précoces. La disponibilité de la radiothérapie et de la chimiothérapie permet d'optimiser une chirurgie adéquate en diminuant le taux de récidive et en améliorant la survie. L'amélioration de l'accès aux soins de qualité et la sensibilisation des populations permettra de réduire la mortalité.

\section{CONFLITS D'INTERET}

Les auteurs n'ont déclaré aucun conflit d'intérêts.

\section{REFERENCES}

I. Collinet P, Poncelet E, Vinatier D. Cancer de l'endomètre. J Gynecol Obstet Biol Reprod 2008 ; $37(2)$ : 57-63.

2. Ouédraogo AS, Sanou-Lamien AM, OuédraogoTiendrébéogo R, Ramde N, Konsegre V, Ido $\mathrm{F}$ et al. Aspects histo-épidémiologiques du cancer de l'endomètre à Ouagadougou. J Afr Cancer 20I I;3(4):25I-255.

3. Diop P. S, Ka I, Ndiaye N, Fall B. Cancers gynécologiques et mammaires à l'Hôpital Général de Grand-Yoff de Dakar : analyse et implications des 
aspects épidémiologiques à propos de 169 cas. J Afr Cancer 2012 ; 4(3): I76-179.

4. Taïeb S, Fauquet I, Narduci F, Ceugnart L. IRM des cancers de l'endomètre. Imagerie de la Femme 2007;17(4):259-263.

5. Kinkel K, Kaji Y, Yu KK, Segal MR, Lu Y, Powell CB et al. Radiologic staging in patients with endometrial cancer: a meta-analysis. Radiology 1999; 2 12(3): 7 II718.

6. Setiawan V. W, Yang H. P, Pike M. C, McCann S. E, Yu H, Xiang Y.B, et al. Type I and II endometrial cancers: have they different risk factors? J Clin Oncol 20I3;3I (20):2607-20I8.

7. Giede C, Le Tien, Power P. Rôle de la chirurgie en matière de cancer de l'endomètre. J Obstet Gynaecol Can 2016;38(I2): SI97-S207.

8. Benedetti Panici P, Basile S, Maneschi F, Alberto Lissoni A, Signorelli M, Scambia G et al. Systematic pelvic lymphadenectomy vs. No lymphadenectomy in early-stage endometrial carcinoma: randomized clinical trial. Natl Cancer Inst 2008;100(23):I7071716.

9. Walker JL, Piedmonte M, Spirtos N, Eisenkop S, Schlaerth J, Mannel RS et al. Surgical staging of uterine cancer : randomized phase Illtrial of laparoscopy versus laparotomy. A gynecologic Oncology Group (GOG): preliminary results. J Clin Oncol 2006 ; 24(I8) : 5010-5010.

10. Lheureux S, Joly F. Cancer de l'endomètre : place de la chimiothérapie adjuvante. Bull Cancer 2012; 99(I):85-9I.

I I. Mazeron R, Monnier L, Belaid A, Berges O, Morice P, Pautier $\mathrm{P}$ et al. Place de la radiothérapie dans la prise en charge postopératoire des patientes atteintes de cancer de l'endomètre. Cancer/Radiothérapie 20II; I5(4):323-329.

12. Iheagwara UK, Vargo JA, Chen KS, Burton DR , Taylor SE, Berger JL. Neoadjuvant Chemoradiation Therapy Followed by Extrafascial Hysterectomy in Locally Advanced Type II Endometrial Cancer Clinically Extending to Cervix. Pract Rad Oncol 2019;9, 248-256.

13. Gauthier T, Siegerth F, Monteil J, Jammet I, Saidi N, Tubiana $\mathrm{N}$ et al. Surveillance du cancer de l'endomètre. Bull Cancer 20 I4; I0 (7-8): 74I-747.

14. Bristow RE, Zerbe MJ, Rosenshein NB, Grumbine FC, Montz FJ. Stage IVB endometrial carcinoma: the role of cytoreductive surgery and determinants of survival. Gynecol Oncol 2000;78(2):85-91.

15. Dem A, Traoré B, Dieng MM, Diop PS, Ouadji T, Lalami MT et al. Les cancers gynécologiques et mammaires à l'Institut du cancer de Dakar, Cahiers Santé 2008. I8(I) :25-29. 